Pacific Journal of Mathematics

FAMILIES OF RIEMANN SURFACES OVER THE PUNCTURED 


\title{
FAMILIES OF RIEMANN SURFACES OVER THE PUNCTURED DISK
}

\author{
Clifford J. Earle and Patricia L. Sipe
}

\begin{abstract}
We study holomorphic families of compact Riemann surfaces over the punctured unit disk. For every genus $p \geq 3$ we define a family whose relative canonical bundle has no roots of order $n>2$. The monodromy group of that family is generated by a product of powers of commuting Dehn twists. We give necessary and sufficient conditions for such a product to generate the monodromy group of a family over the punctured disk.
\end{abstract}

1. Introduction. By definition an $n$th root (or root of order $n$ ) of a holomorphic line bundle $K$ over a complex manifold $M$ is a holomorphic line bundle $L \rightarrow M$ such that the line bundles $L^{\otimes n} \rightarrow M$ and $K \rightarrow M$ are equivalent. (Every line bundle in this paper will be holomorphic, and equivalence will always be holomorphic equivalence.) In this paper, which continues the study initiated by the second author in [15] and [17], we shall be interested primarily in $n$th roots of the relative canonical bundle $K_{\text {rel }}(V) \rightarrow V$ of a holomorphic family $\pi: V \rightarrow B$ of compact Riemann surfaces $p \geq 2$. Readers who are unfamiliar with holomorphic families and their relative canonical bundles can find the basic facts about them in $\S 1$ of [17]. The most important feature of $K_{\text {rel }}(V) \rightarrow V$ is that its restriction to each fiber $X_{t}=\pi^{-1}(t) \subset V$ is the canonical line bundle of the Riemann surface $X_{t}$.

For every genus $p \geq 3$, examples were given in [17] of holomorphic families whose relative canonical bundles have no roots of order $n>$ 2 . The base space of each of these families is a quotient space of the Teichmüller space $T_{p}$ of compact Riemann surfaces of genus $p$, and has complex dimension $3 p-3$. The simplest base space for such examples would be the punctured unit disk

$$
\Delta^{\prime}=\{z \in \mathbb{C} ; 0<|z|<1\} .
$$

This paper presents examples with base space $\Delta^{\prime}$ for every genus $p \geq$ 3 . We define the required families in the next section.

To prove Theorem 1, which states that the relative canonical bundles of these families have no roots of order $n>2$, we study their 
monodromy groups in $\S \S 4$ and 6 . Because it is a subgroup of the Teichmüller modular group, the monodromy group of a family acts on the set $E_{n}$ of equivalence classes of $n$th roots of the relative canonical bundle of the Teichmüller curve (see $\S \S 3$ and 5). Theorem 2 in $\S 5$ uses that action to give a necessary condition for the existence of $n$th roots. The relative canonical bundle of a family cannot have a root of order $n$ unless the monodromy group leaves some point of $E_{n}$ fixed.

Among the simplest elements of the modular group that act without fixed points on every $E_{n}$ with $n>2$ are products of opposite Dehn twists about two homologous curves (like $A_{2}$ and $D_{2}$ in Figure 1c) that separate the surface into two pieces of positive genus; see [15] and [17] for details. We first tried to construct families over $\Delta^{\prime}$ whose monodromy groups are generated by such elements. To our surprise we found that task impossible and had to make a slight adjustment in our strategy (see Remark 1 in $\S 6$ ). The obstruction to our first plan is described by Theorem 3 in $\S 7$, which gives necessary and sufficient conditions for a product of powers of commuting Dehn twists to generate the monodromy of a family over $\Delta^{\prime}$. Theorem 3 is a special case of a more general result (see Remark 1 in $\S 7$ ) that characterizes all elements of the modular group that generate the monodromy of some family over $\Delta^{\prime}$. That result will be proved in our forthcoming paper [7].

We are grateful to Claude LeBrun for pointing out an error in our original formulation of Theorem 2. In addition, the second author thanks the Mathematics Department at Universidad Técnica Federico Santa Maria in Valparaíso, Chile, for its hospitality during the 198788 academic year, when this research was completed.

2. The examples. Given $p \geq 3$, choose compact Riemann surfaces $X$ and $Y$ of genus 1 and $p-2$ respectively. Choose distinct points $P_{1}, P_{2}$ on $X$ and $Q_{1}, Q_{2}$ on $Y$, disjoint open neighborhoods $U_{1}, U_{2} \subset X$ and $V_{1}, V_{2} \subset Y$ of these points (see Figure 1a), and local coordinates $z_{1}, z_{2}$ and $w_{1}, w_{2}$ that map these neighborhoods onto the open unit disk and satisfy

$$
z_{1}\left(P_{1}\right)=z_{2}\left(P_{2}\right)=w_{1}\left(Q_{1}\right)=w_{2}\left(Q_{2}\right)=0 .
$$

Using a standard construction (cf. p. 37 of [8]) we define a holomorphic family $\pi: W \rightarrow \Delta^{\prime}$ as follows. Let

(2.1a) $X^{*}=\left\{(t, P) \in \Delta^{\prime} \times X ;\left|z_{1}(P)\right|>|t|\right.$ if $P \in U_{1}$ and

$$
\left.\left|z_{2}(P)\right|>|t|^{2 p-3} \text { if } P \in U_{2}\right\}
$$



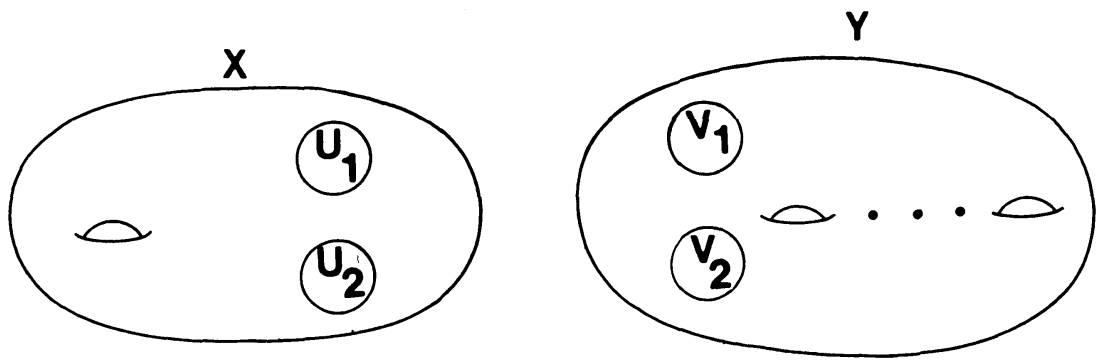

Figure 1a
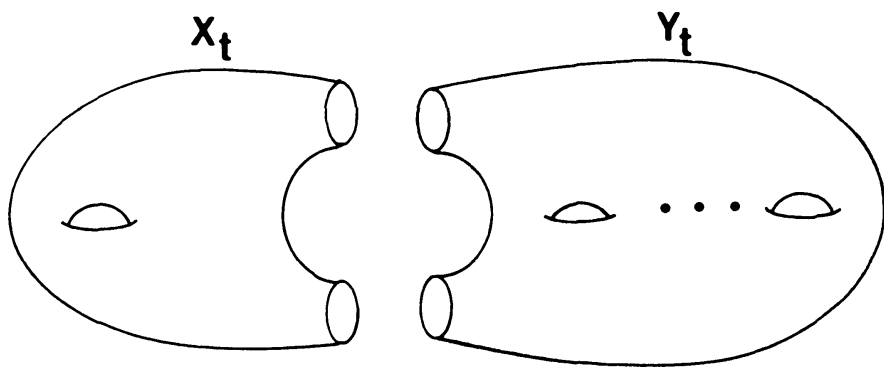

FIGURE 1b

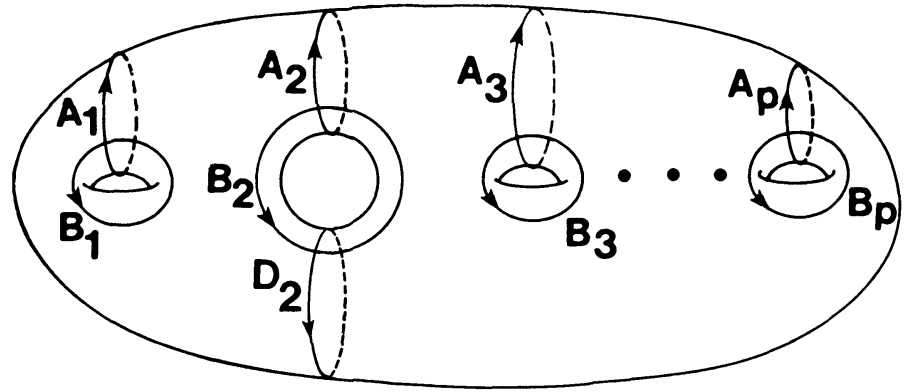

FIGURE 1c

and

(2.1b) $Y^{*}=\left\{(t, Q) \in \Delta^{\prime} \times Y ;\left|w_{1}(Q)\right|>|t|\right.$ if $Q \in V_{1}$ and

$$
\left.\left|w_{2}(Q)\right|>|t|^{2 p-3} \text { if } Q \in V_{2}\right\} .
$$

The complex manifold $W$ is obtained from $X^{*} \cup Y^{*}$ by identifying $(s, P) \in X^{*}$ with $(t, Q) \in Y^{*}$ if $s=t$ and either

$$
P \in U_{1}, \quad Q \in V_{1}, \quad \text { and } \quad z_{1}(P) w_{1}(Q)=t
$$

or

$$
P \in U_{2}, \quad Q \in V_{2}, \quad \text { and } \quad z_{2}(P) w_{2}(Q)=t^{2 p-3} .
$$


The projections $(t, P) \mapsto t$ and $(t, Q) \mapsto t$ from $X^{*}$ and $Y^{*}$ to $\Delta^{\prime}$ induce a well-defined holomorphic map $\pi: W \rightarrow \Delta^{\prime}$, which defines a holomorphic family of compact Riemann surfaces of genus $p$. Our principal result is

THEOREM 1. The relative canonical bundle of the family $\pi: W \rightarrow \Delta^{\prime}$ defined above has no roots of order $n>2$.

We shall prove Theorem 1 in $\S 6$. The proof will rely on the results in $\S 5$ about the action of the Teichmüller modular group on roots of order $n$. The necessary background material about the Teichmüller curve, the modular group, and monodromy is presented in $\S \S 3$ and 4.

3. The modular group and the Teichmüller curve. The material in this section and the next is well known. The primary reference is [9]; see also Chapter 5 of Nag [14]. For any integer $p \geq 2$ the Teichmüller curve $\pi_{p}: V_{p} \rightarrow T_{p}$ (see Nag [14] or $\S 2$ of [11]) is a holomorphic family of compact Riemann surfaces of genus $p$. Let $S$ be any smooth oriented compact surface of genus $p$. Since the Teichmüller space $T_{p}$ is smoothly contractible, there is a diffeomorphism $\theta: T_{p} \times S \rightarrow V_{p}$ such that $\pi_{p}(\theta(t, x))=t$ for all $(t, x)$ in $T_{p} \times S$. Since each fiber $\pi_{p}^{-1}(t)$ has a natural orientation induced by its complex structure, we can and do require that the diffeomorphisms $x \mapsto \theta(t, x)$ from $S$ to $\pi_{p}^{-1}(t)$ respect the given orientations. For a simple construction of $\theta$, see [4].

Let $\mathscr{H}^{+}(S)$ be the group of sense-preserving homeomorphisms of $S$ onto itself. By definition, the Teichmüller modular group $\operatorname{Mod}_{p}$ is $\mathscr{H}^{+}(S)$ modulo the normal subgroup of homeomorphisms that are isotopic to the identity. The diffeomorphism $\theta$ above leads to an identification between $\operatorname{Mod}_{p}$ and the automorphism group of the Teichmüller curve, which we shall now describe.

By definition, a map of holomorphic families from the family $\pi^{\prime}$ : $V^{\prime} \rightarrow B^{\prime}$ to the family $\pi: V \rightarrow B$ is a holomorphic map $\tilde{f}: V^{\prime} \rightarrow V$ with the following two properties:

(i) there is a (necessarily holomorphic) map $f: B^{\prime} \rightarrow B$ such that $f \circ \pi^{\prime}=\pi \circ \tilde{f}$

(ii) for each $t$ in $B^{\prime}, \tilde{f}$ restricts to a bijective map from $\left(\pi^{\prime}\right)^{-1}(t)$ to $\pi^{-1}(f(t))$.

The map of holomorphic families $\tilde{f}: V^{\prime} \rightarrow V$ is an isomorphism if $\tilde{f}$ (and therefore $f$ ) is biholomorphic. An isomorphism is an automorphism if $V^{\prime}=V, B^{\prime}=B$, and $\pi^{\prime}=\pi$. 
If $\tilde{f}: V_{p} \rightarrow V_{p}$ is an automorphism of the Teichmüller curve, the diffeomorphism $\theta^{-1} \circ \tilde{f} \circ \theta: T_{p} \times S \rightarrow T_{p} \times S$ is fiber preserving. Therefore, for each $t$ in $T_{p}$ there is a diffeomorphism $f_{t}$ in $\mathscr{Z}^{+}(S)$ such that

$$
\tilde{f}(\theta(t, x))=\theta\left(f(t), f_{t}(x)\right) \quad \text { for all }(t, x) \in T_{p} \times S .
$$

The image $\left[f_{t}\right]$ of $f_{t}$ in $\operatorname{Mod}_{p}$ is independent of $t$, so formula (3.1) determines a homomorphism $\tilde{f} \mapsto\left[f_{t}\right]$ from the automorphism group of the Teichmüller curve to $\operatorname{Mod}_{p}$. That homomorphism is a surjective isomorphism (see [9] or Corollary 2 on p. 265 of [5]) and provides the desired identification of $\operatorname{Mod}_{p}$ with the automorphism group.

4. The monodromy of a holomorphic family. Let $\pi: V \rightarrow B$ be any holomorphic family of compact Riemann surfaces of genus $p \geq 2$. We recall the definition of its monodromy. Choose a basepoint $b_{0}$ in $B$ and a homeomorphism $\eta_{0}: S \rightarrow \pi^{-1}\left(b_{0}\right)$ that respects the given orientations. Let $I=[0,1]$. If $h: I \rightarrow B$ is any loop with $h(0)=$ $h(1)=b_{0}$, choose a continuous map $\eta: I \times S \rightarrow V$ such that

$$
\pi(\eta(u, x))=h(u) \text { for all }(u, x) \in I \times S,
$$

the map $\eta(u, \cdot)$ from $S$ to $\pi^{-1}(h(u))$ is a homeomorphism for each $u$ in $I$, and $\eta(0, \cdot)$ is the homeomorphism $\eta_{0}$ chosen above. We set $\eta_{u}=\eta(u, \cdot)$ for each $u$ in $I$. It is well known and easy to prove that the isotopy class of the homeomorphism $\eta_{1}^{-1} \circ \eta_{0}$ of $S$ onto itself depends only on the homotopy class $\gamma$ of the loop $h$ (and the isotopy class of $\eta_{0}$ ), and that

$$
\gamma \mapsto \mu(\gamma)=\left[\eta_{1}^{-1} \circ \eta_{0}\right]
$$

is a homomorphism of the fundamental group $\Gamma=\pi_{1}\left(B, b_{0}\right)$ into $\operatorname{Mod}_{p}$. We call $\mu$ the monodromy homomorphism of the family $\pi: V \rightarrow B$; its monodromy group is the subgroup $\mu(\Gamma)$ of $\operatorname{Mod}_{p}$.

Now let $\omega: B^{\#} \rightarrow B$ be a holomorphic universal covering map, and let $\pi^{\#}: V^{\#} \rightarrow B^{\#}$ be the pullback of $\pi: V \rightarrow B$ by $\omega$. Thus

$$
V^{\#}=\omega^{-1}(V)=\left\{(t, v) \in B^{\#} \times V ; \omega(t)=\pi(v)\right\}
$$

and $\pi^{\#}(t, v)=t$. We identify $\Gamma=\pi_{1}\left(B, b_{0}\right)$ with the group of cover transformations $\gamma: B^{\#} \rightarrow B^{\#}$ of $B^{\#}$ over $B$ in the usual way, by choosing a point $t_{0}$ in $B^{\#}$ with $\omega\left(t_{0}\right)=b_{0}$. Each $\gamma$ in $\Gamma$ then induces an automorphism $\tilde{\gamma}$ of the family $\pi^{\#}: V^{\#} \rightarrow B^{\#}$, given by

$$
\tilde{\gamma}(t, v)=(\gamma(t), v) \quad \text { for all } \gamma \in \Gamma \text { and }(t, v) \in V^{\#} \text {. }
$$


Since $B^{\#}$ is simply connected, the homeomorphism $x \mapsto\left(t_{0}, \eta_{0}(x)\right)$ of $S$ onto $\left(\pi^{\#}\right)^{-1}\left(t_{0}\right)$ determines a "marking", or "structure of Teichmüller" (see $\S 2$ of [9] or Chapter 5 of [14]) on the family $\pi^{\# \text { : }}$ $V^{\#} \rightarrow B^{\#}$. Similarly, the diffeomorphism $\theta: T_{p} \times S \rightarrow V_{p}$ of $\S 3$ determines such a structure on the Teichmüller curve $\pi_{p}: V_{p} \rightarrow T_{p}$. By Theorem 3.1 of [9] (or Theorem 5.4.3 of [14]) there is a unique map of holomorphic families $\tilde{f}: V^{\#} \rightarrow V_{p}$ such that the homeomorphisms

$$
x \mapsto \tilde{f}\left(t_{0}, \eta_{0}(x)\right) \quad \text { and } \quad x \mapsto \theta\left(f\left(t_{0}\right), x\right)
$$

of $S$ onto $\pi_{p}^{-1}\left(f\left(t_{0}\right)\right)$ are isotopic. The uniqueness of $\tilde{f}$ implies the important formula

$$
\tilde{f} \circ \tilde{\gamma}=\mu(\gamma) \circ \tilde{f} \text { for all } \gamma \in \Gamma .
$$

For the reader's convenience we shall outline a proof of (4.4). Put $t_{1}=\gamma\left(t_{0}\right)$ and choose a path $u \mapsto t_{u}, u \in I \quad(=[0,1])$, from $t_{0}$ to $t_{1}$ in $B^{\#}$. The loop $u \mapsto \omega\left(t_{u}\right)$ in $B$ belongs to the homotopy class $\gamma$ in $\Gamma$. For each $u$ in $I$ let $\varphi_{u}$ be the unique homeomorphism of $S$ onto $\pi^{-1}\left(\omega\left(t_{u}\right)\right)$ such that

$$
\tilde{f}\left(t_{u}, \varphi_{u}(x)\right)=\theta\left(f\left(t_{u}\right), x\right) \text { for all } x \in S .
$$

Then $\mu(\gamma)=\left[\varphi_{1}^{-1} \circ \varphi_{0}\right]$ by the definition of monodromy, since $\varphi_{0}$ and $\eta_{0}$ are isotopic.

Let $\tilde{g}: V^{\#} \rightarrow V_{p}$ be the map of holomorphic families $\mu(\gamma)^{-1} \circ \tilde{f} \circ \tilde{\gamma}$. We must show that the homeomorphisms

$$
x \mapsto \tilde{g}\left(t_{0}, \eta_{0}(x)\right) \quad \text { and } \quad x \mapsto \theta\left(g\left(t_{0}\right), x\right)
$$

from $S$ to $\pi_{p}^{-1}\left(g\left(t_{0}\right)\right)$ are isotopic (so that $\tilde{g}=\tilde{f}$ as required). Now (4.3) and (4.5) imply that

$$
(\mu(\gamma) \circ \tilde{g})\left(t_{0}, \eta_{0}(x)\right)=\tilde{f}\left(t_{1}, \eta_{0}(x)\right)=\theta\left(f\left(t_{1}\right),\left(\varphi_{1}^{-1} \circ \eta_{0}\right)(x)\right) .
$$

In addition, by (3.1) we can write

$$
(\mu(\gamma) \circ \theta)\left(g\left(t_{0}\right), x\right)=\theta\left(f\left(t_{1}\right), \psi(x)\right),
$$

with $\psi \in \mathscr{H}^{+}(S)$ and $[\psi]=\mu(\gamma)$. The desired isotopy now follows from the fact that $\mu(\gamma)=\left[\varphi_{1}^{-1} \circ \varphi_{0}\right]=\left[\varphi_{1}^{-1} \circ \eta_{0}\right]$.

REMARK. Formula (4.4) makes it possible to reconstruct the family $\pi: V \rightarrow B$ from the map $f: B^{\#} \rightarrow T_{p}$ and the monodromy homomorphism $\mu$ (cf. Theorem 4.1 of [9]). 
5. Monodromy and $n$th roots. The action of $\operatorname{Mod}_{p}$ as a group of automorphisms $\tilde{f}: V_{p} \rightarrow V_{p}$ of the Teichmüller curve induces an action on line bundles $L \rightarrow V_{p}$ by pullback:

$$
\tilde{f} \cdot L=\left(\tilde{f}^{-1}\right)^{*}(L) \text { for all } \tilde{f} \in \operatorname{Mod}_{p} .
$$

Since $\left(\tilde{f}^{-1}\right)^{*} K_{\mathrm{rel}}\left(V_{p}\right)$ and $K_{\mathrm{rel}}\left(V_{p}\right)$ are equivalent (see Proposition 1 of [17]) and pullbacks preserve tensor products, (5.1) determines an action of $\operatorname{Mod}_{p}$ on the finite set $E_{n}$ of equivalence classes of $n$th roots of $K_{\text {rel }}\left(V_{p}\right)$ for each $n \geq 2$. This action on $E_{n}$ can be used to study $n$th roots of $K_{\text {rel }}(V)$ for any holomorphic family of closed Riemann surfaces $\pi: V \rightarrow B$.

THEOREM 2. The map of holomorphic families $\tilde{f}$ in formula (4.4) induces a map from the set of equivalence classes of nth roots of $K_{\mathrm{rel}}(V)$ into the set of elements of $E_{n}$ that are fixed by every member of the monodromy group.

We shall describe that map in $\S 8$ when we prove Theorem 2.

To use the theorem effectively we must know how to compute the action of $\operatorname{Mod}_{p}$ on $E_{n}$. We recall some results from [15]. Let $T_{0}(S)$ be the bundle of non-zero tangent vectors to the smooth surface $S$ of genus $p$. For each $\tilde{f}$ in $\operatorname{Mod}_{p}$, let $\sigma_{n}(\tilde{f})$ be the automorphism of $H_{1}\left(T_{0}(S), Z_{n}\right)$ induced by (any diffeomorphism of $S$ that represents) $\tilde{f}$. Let $\alpha$ be the homology class in $H_{1}\left(T_{0}(S), Z_{n}\right)$ determined by the positive (counterclockwise) loop around the origin in the fiber $\mathbb{R}^{2} \backslash$ origin $\}$ of $T_{0}(S)$, and let $\Phi_{n}$ be the set of all homomorphisms $\varphi: H_{1}\left(T_{0}(S), Z_{n}\right) \rightarrow Z_{n}$ such that $\varphi(\alpha)=-1$. Then $\operatorname{Mod}_{p}$ acts on $\Phi_{n}$ by

$$
\tilde{f} \cdot \varphi=\varphi \circ \sigma_{n}(\tilde{f})^{-1} \quad \text { for all } \tilde{f} \in \operatorname{Mod}_{p} .
$$

Formula (2a) in Proposition 2.2 of [15] shows that $K_{\text {rel }}\left(V_{p}\right)$ and the canonical bundle $K\left(V_{p}\right)$ are equivalent, so Theorem 1 of [15] provides a natural bijection between the sets $E_{n}$ and $\Phi_{n}$, which is equivariant with respect to the actions (5.1) and (5.2) of $\operatorname{Mod}_{p}$. That result is very useful because it is easy to determine the set $\Phi_{n}$ and some important automorphisms $\sigma_{n}(\tilde{f})$. For instance it is well known (see Proposition 2.5 of [15]) that $(2 p-2) \alpha=0$, so $\Phi_{n}$ is empty unless (as we shall assume for the remainder of this section) $n$ divides $2 p-2$.

The automorphisms $\sigma_{n}(\tilde{f})$ are described by their matrices with respect to a basis for the group $H_{1}\left(T_{0}(S), Z_{n}\right)$, which is isomorphic to $\left(Z_{n}\right)^{2 p+1}$ since $n$ divides $2 p-2$ (see p. 74 of [15]). To obtain a 
convenient basis we choose a Riemannian metric on $S$ and smooth simple loops $\left\{A_{1}, \ldots, A_{p}, B_{1}, \ldots, B_{p}\right\}$ in $S$ that define a canonical basis for $H_{1}(S, Z)$, as illustrated in Figure 1c. We also choose a diffeomorphism $r_{p}: S \rightarrow S$ of order $p$ that satisfies $r_{p}\left(A_{p}\right)=A_{1}$, $r_{p}\left(B_{p}\right)=B_{1}$, and $r_{p}\left(A_{i}\right)=A_{i+1}$ and $r_{p}\left(B_{i}\right)=B_{i+1}$ if $1 \leq i \leq p-1$.

If $\gamma$ is a smooth loop in $S$, parametrize $\gamma$ by arc length and let $\bar{\gamma}$ be the homology class in $H_{1}\left(T_{0}(S), Z_{n}\right)$ of the smooth loop $t \mapsto$ $\left(\gamma(t), \gamma^{\prime}(t)\right)$ in $T_{0}(S)$. The collection

$$
\left\{\bar{A}_{1}+\alpha, \bar{A}_{2}+\alpha, \ldots, \bar{A}_{p}+\alpha, \bar{B}_{1}+\alpha, \ldots, \bar{B}_{p}+\alpha, \alpha\right\}
$$

is the desired basis for $H_{1}\left(T_{0}(S), Z_{n}\right)$.

For any simple loop $\gamma$ in $S$, let $f_{\gamma}$ denote the positive Dehn twist (turn right at $\gamma$ ) about $\gamma$. If $C_{1}$ is a simple loop such that $A_{1} C_{1}$ is freely homotopic to $A_{2}$, then $\left[f_{A_{1}}\right],\left[f_{B_{1}}\right],\left[f_{C_{1}}\right]$, and $\left[r_{p}\right]$ generate $\operatorname{Mod}_{p}$ (see pp. 76 and 77 of [15]). Our first proposition describes $\sigma_{n}(\tilde{f})$ for these generators. We must introduce some notation. We write

$e_{i}=$ the row vector in $\left(Z_{n}\right)^{p}$ with $i$ th entry 1 , all other entries zero, $E_{i}=$ the $p \times p$ matrix with $i$ th row $e_{i}$, all other rows zero,

$I=$ the $p \times p$ identity matrix,

$R=\left(r_{i j}\right)=$ the $p \times p$ permutation matrix with

$$
\begin{aligned}
& r_{i j}=\delta_{i, j+1} \text { if } 1 \leq j \leq p-1, \\
& r_{i p}=\delta_{i, 1},
\end{aligned}
$$

$M=\left(m_{i j}\right)=$ the $p \times p$ matrix with $m_{11}=m_{22}=1$,

$$
m_{12}=m_{21}=-1 \text {, all other entries zero. }
$$

Proposition 5.1. With respect to the basis (5.3) for $H_{1}\left(T_{0}(S), Z_{n}\right)$ we have

$$
\begin{aligned}
& \sigma_{n}\left(\left[f_{A_{1}}\right]\right)=\left(\begin{array}{ccc}
I & E_{1} & 0 \\
0 & I & 0 \\
0 & -e_{1} & 1
\end{array}\right), \quad \sigma_{n}\left(\left[f_{B_{1}}\right]\right)=\left(\begin{array}{ccc}
I & 0 & 0 \\
-E_{1} & I & 0 \\
e_{1} & 0 & 1
\end{array}\right), \\
& \sigma_{n}\left(\left[f_{C_{1}}\right]\right)=\left(\begin{array}{ccc}
I & M & 0 \\
0 & I & 0 \\
0 & \left(e_{1}-e_{2}\right) & 1
\end{array}\right), \quad \sigma_{n}\left(\left[r_{p}\right]\right)=\left(\begin{array}{ccc}
R & 0 & 0 \\
0 & R & 0 \\
0 & 0 & 1
\end{array}\right) .
\end{aligned}
$$

Proof. Proposition 3.2 of [15] gives the matrices of these automorphisms with respect to a slightly different basis for $H_{1}\left(T_{0}(S), Z_{n}\right)$. Conjugation of those matrices by the matrix for the change of basis gives the matrices above. 
The proof of Theorem 1 will rely on the following proposition and its corollaries.

Proposition 5.2. Let $A_{2}$ and $D_{2}$ be the simple loops illustrated in Figure 1c on the surface of genus $p \geq 3$. The matrix of $\sigma_{n}\left(\left[\left(f_{D_{2}}\right)^{2 p-3} \circ f_{A_{2}}\right]\right)$ with respect to the basis (5.3) is

$$
\left(\begin{array}{ccc}
I & 0 & 0 \\
0 & I & 0 \\
0 & 2 e_{2} & 1
\end{array}\right)
$$

Proof. Since $\left[r_{p} \circ f_{A_{1}}\right]=\left[f_{A_{2}} \circ r_{p}\right]$, Proposition 5.1 implies

$$
\sigma_{n}\left(\left[f_{A_{2}}\right]\right)=\left(\begin{array}{ccc}
I & E_{2} & 0 \\
0 & I & 0 \\
0 & -e_{2} & 1
\end{array}\right) \text {. }
$$

According to p. 520 of [16],

$$
\sigma_{n}\left(\left[\left(f_{D_{2}}\right)^{-1} \circ f_{A_{2}}\right]\right)=\left(\begin{array}{ccc}
I & 0 & 0 \\
0 & I & 0 \\
0 & 2 e_{2} & 1
\end{array}\right) .
$$

An easy calculation now establishes the proposition.

Corollary 5.3. If $p \geq 3$ and $n>2$, the element

$$
\tilde{f}=\left[f_{A_{2}}^{-1} \circ\left(f_{D_{2}}\right)^{-(2 p-3)}\right]
$$

of $\operatorname{Mod}_{p}$ has no fixed points in $\Phi_{n}$.

Proof. Consider the basis element $\widetilde{B}_{2}=\bar{B}_{2}+\alpha$ in $H_{1}\left(T_{0}(S), Z_{n}\right)$. If $\varphi \in \Phi_{n},(5.2)$ and (5.4) give

$$
(\tilde{f} \cdot \varphi)\left(\widetilde{B}_{2}\right)=\varphi\left(\sigma_{n}\left(\tilde{f}^{-1}\right)\left(\widetilde{B}_{2}\right)\right)=\varphi\left(\widetilde{B}_{2}+2 \alpha\right)=\varphi\left(\widetilde{B}_{2}\right)-2,
$$

so $\tilde{f} \cdot \varphi \neq \varphi$.

As an immediate consequence of Corollary 5.3 and Theorem 2 we have

Corollary 5.4. Let $\pi: V \rightarrow B$ be a holomorphic family of compact Riemann surfaces of genus $p \geq 3$. If $\left[f_{A_{2}}^{-1} \circ\left(f_{D_{2}}\right)^{-(2 p-3)}\right]$ belongs to the monodromy group of the family, then the relative canonical bundle has no roots of order $n>2$.

REMARKS. (1) In Corollaries 5.3 and 5.4 we tacitly assumed that $n$ divides $2 p-2$. If $n$ does not divide $2 p-2$, both corollaries are vacuously true. 
(2) The matrices in Proposition 5.1 are described indirectly on p. 523 of [16], in which the reasons for using the basis (5.3) are explained.

6. Proof of Theorem 1. We must prove that $K_{\text {rel }}(W)$ has no roots of order $n>2$ if $\pi: W \rightarrow \Delta^{\prime}$ is the family of genus $p \geq 3$ defined in $\S 2$. By Corollary 5.4 , it suffices to prove that $\left[f_{A_{2}}^{-1} \circ\left(f_{D_{2}}\right)^{-(2 p-3)}\right]$ belongs to the monodromy group. For that purpose we must study the geometry of the family. We use the notation of $\S 2$. In addition, for each $t$ in $\Delta^{\prime}$ we put

(6.1a) $X_{t}=\left\{P \in X ;\left|z_{1}(P)\right|^{2} \geq|t|\right.$ if $P \in U_{1}$ and

$$
\left.\left|z_{2}(P)\right|^{2} \geq|t|^{2 p-3} \text { if } P \in U_{2}\right\}
$$

and

(6.1b) $Y_{t}=\left\{Q \in Y ;\left|w_{1}(Q)\right|^{2} \geq|t|\right.$ if $Q \in V_{1}$ and

$$
\left.\left|w_{2}(Q)\right|^{2} \geq|t|^{2 p-3} \text { if } Q \in V_{2}\right\}
$$

and we define $S_{t}$ to be the compact Riemann surface of genus $p$ obtained from $X_{t} \cup Y_{t}$ by attaching $\partial X_{t}$ and $\partial Y_{t}$ (see Figures $1 \mathrm{~b}$ and 1c) so that $P$ in $\partial X_{t}$ and $Q$ in $\partial Y_{t}$ are identified when either

(6.2a) $\quad P \in U_{1}, \quad Q \in V_{1}, \quad$ and $z_{1}(P) w_{1}(Q)=t$

or

(6.2b) $\quad P \in U_{2}, \quad Q \in V_{2}, \quad$ and $\quad z_{2}(P) w_{2}(Q)=t^{2 p-3}$.

Comparing (6.1) and (6.2) with (2.1) and (2.2) we see that the map $P \mapsto(t, P)$ from $X_{t} \cup Y_{t}$ into $X^{*} \cup Y^{*}$ induces a conformal map of $S_{t}$ onto the fiber $\pi^{-1}(t)$ in $W$.

Observe that $X_{t}$ and $Y_{t}$ depend only on the absolute value of $t$. The argument of $t$ determines how the boundaries $\partial X_{t}$ and $\partial Y_{t}$ are attached to each other.

To compute the monodromy, fix $r$ in the open interval $(0,1)$ and let $\gamma$ be the generator of $\pi_{1}\left(\Delta^{\prime}, r\right)$ represented by the loop

$$
h(u)=r e^{2 \pi i u}, \quad u \in I=[0,1] .
$$

Let $A_{2}$ and $D_{2}$ be the respective images in $S_{r}$ of the boundary curves $\left|w_{1}(Q)\right|^{2}=r$ and $\left|w_{2}(Q)\right|^{2}=r^{2 p-3}$ in $Y_{r}$ (see Figures $1 \mathrm{~b}$ and $1 \mathrm{c}$ ). Theorem 1 follows at once from Corollary 5.4 and

LEMMA 6.1. For each $p \geq 3$, the monodromy group of the family $\pi: W \rightarrow \Delta^{\prime}$ defined in $\S 2$ is generated by

$$
\mu(\gamma)=\left[f_{A_{2}}^{-1} \circ\left(f_{D_{2}}\right)^{-(2 p-3)}\right]
$$


Proof. Choose $s$ with $r<s<1$. We shall produce a family of homeomorphisms $\eta_{u}: S_{r} \rightarrow \pi^{-1}(h(u)) \subset W$, depending continuously on $u$ in $I$, by mapping $X_{r}$ into $X^{*}$ and $Y_{r}$ into $Y^{*}$ in such a way that

$$
\eta_{u}(P)=(h(u), P) \quad \text { if } P \in X_{r}
$$

and

$$
\eta_{u}(Q)=(h(u), Q) \text { if } Q \in Y_{s} .
$$

It remains to define $\eta_{u}$ in $Y_{r} \backslash Y_{s}$, which is the union of the disjoint annuli

$$
R_{1}=\left\{Q \in V_{1} ; r \leq\left|w_{1}(Q)\right|^{2}<s\right\}
$$

and

$$
R_{2}=\left\{Q \in V_{2} ; r^{2 p-3} \leq\left|w_{2}(Q)\right|^{2}<s^{2 p-3}\right\}
$$

However that is done, $\eta_{1}^{-1} \circ \eta_{0}$ will be the identity outside $R_{1} \cup R_{2}$, so it must be the composition of a power of $f_{A_{2}}$ and a power of $f_{D_{2}}$. To determine these powers, observe that in $R_{j}, j=1$ or $2, \eta_{u}$ has the form

$$
\eta_{u}(Q)=\left(h(u), \varphi_{u}(Q)\right)
$$

where $\varphi_{u}$ is a homeomorphism of $R_{j}$ onto itself. Formulas (6.3) and the identifications (2.2) and (6.2) determine the boundary values of $\varphi_{u}$. For $j=1$ the boundary conditions are

$$
w_{1}\left(\varphi_{u}(Q)\right)=w_{1}(Q) \text { when }\left|w_{1}(Q)\right|^{2}=s
$$

and

$$
w_{1}\left(\varphi_{u}(Q)\right)=e^{2 \pi i u} w_{1}(Q) \text { when }\left|w_{1}(Q)\right|^{2}=r .
$$

It is easy to extend $\varphi_{u}$ to $R_{1}$ so that these boundary conditions are satisfied and $\varphi_{u}$ depends continuously on $u$. When that is done we find that

$$
\eta_{1}^{-1} \circ \eta_{0}=\varphi_{1}^{-1} \circ \varphi_{0}=f_{A_{2}}^{-1} \text { in } R_{1} \text {. }
$$

Similarly, $\eta_{1}^{-1} \circ \eta_{0}=\left(f_{D_{2}}\right)^{-(2 p-3)}$ in $R_{2}$, so

$$
\mu(\gamma)=\left[\eta_{1}^{-1} \circ \eta_{0}\right]=\left[f_{A_{2}}^{-1} \circ\left(f_{D_{2}}\right)^{-(2 p-3)}\right] .
$$

REMARKS. (1) Our first plan was to construct a family with a monodromy group generated by $\left[f_{A_{2}}^{-1} \circ f_{D_{2}}\right]$. Theorem 3 in $\S 7$ explains why this plan failed; it also suggested to us a successful modification. Since $f_{D_{2}}^{-(2 p-2)}$ induces the identity map on $H_{1}\left(T_{0}(S), Z_{n}\right)$, the composition

$$
\mu(\gamma)=\left[f_{A_{2}}^{-1} \circ f_{D_{2}}\right] \circ\left[f_{D_{2}}^{-(2 p-2)}\right]=\left[f_{A_{2}}^{-1} \circ f_{D_{2}}^{-(2 p-3)}\right]
$$


acts on $E_{n}$ in the same way as $\left[f_{A_{2}}^{-1} \circ f_{D_{2}}\right]$. The exponents of $f_{A_{2}}$ and $f_{D_{2}}$ in $\mu(\gamma)$ are both negative, so we were able to construct families whose monodromy groups are generated by $\mu(\gamma)$.

(2) Theorem 1 asserts that the relative canonical bundles of our examples have no roots of order $n>2$, but says nothing about square roots. The situation for square roots is quite different. Indeed, according to Theorem 4 of Atiyah [1], the relative canonical bundle of any holomorphic family of compact Riemann surfaces of genus $p \geq 2$ over the punctured unit disk has at least one square root. In the case of our families $\pi: W \rightarrow \Delta^{\prime}$ every square root of $K_{\text {rel }}\left(V_{p}\right)$ corresponds to a square root of $K_{\text {rel }}(W)$. That is true because the matrix (5.4) reduces to the identity when $n=2$, and the map in Theorem 2 is bijective when $B=\Delta^{\prime}$ (see the remark at the end of $\S 8$ ).

7. Characterizing some monodromy homomorphisms. Our final theorem places new restrictions on the monodromy of a holomorphic family over $\Delta^{\prime}$. As in $\S 6$, let $\gamma$ be the generator of $\pi_{1}\left(\Delta^{\prime}\right)$ determined by the positive loop around the origin in $\Delta^{\prime}$.

THEOREM 3. Let $\tau_{1}, \ldots, \tau_{k}$ in $\operatorname{Mod}_{p}$ correspond to the negative Dehn twists about $k$ homotopically independent simple loops on the surface $S$ of genus $p \geq 2$. A necessary and sufficient condition for the existence of a holomorphic family $\pi: V \rightarrow \Delta^{\prime}$ of compact Riemann surfaces of genus $p$ whose monodromy homomorphism $\mu$ satisfies

$$
\mu(\gamma)=\tau_{1}^{n_{1}} \tau_{2}^{n_{2}} \cdots \tau_{k}^{n_{k}}
$$

is that all the integers $n_{j}$ be non-negative.

Proof. The sufficiency is easy. If all $n_{j}=0$, any trivial family $\Delta^{\prime} \times X \rightarrow \Delta^{\prime}$ has the required monodromy. If some $n_{j}$ are positive and none are negative, a construction like the one in $\S 2$ produces a family with the desired property. We leave the details to the reader.

To prove the necessity we use some special coordinate functions on the Teichmüller space $T_{p}$. Let $U$ be the upper half plane in $\mathbb{C}$. According to Kra [12] (see also [2], [6], and [13]) there is a holomorphic embedding $\Phi: T_{p} \rightarrow \mathbb{C}^{3 p-3}$ with the following two properties:

$$
\begin{gathered}
\Phi\left(T_{p}\right) \subset U^{3 p-3}, \\
\Phi\left(\tau_{j}(t)\right)=\Phi(t)+e_{j} \quad \begin{array}{l}
\text { for all } t \in T_{p} \text { and } \\
1 \leq j \leq k(\leq 3 p-3) .
\end{array}
\end{gathered}
$$


Here as usual $e_{1}, \ldots, e_{3 p-3}$ are the standard basis vectors for $\mathbb{C}^{3 p-3}$, and $\tau_{j}: T_{p} \rightarrow T_{p}$ is the biholomorphic map induced by $\tau_{j} \in \operatorname{Mod}_{p}$.

Let $\omega: U \rightarrow \Delta^{\prime}$ be the universal covering map

$$
\omega(z)=\exp (2 \pi i z),
$$

so that $\gamma$ in $\pi_{1}\left(\Delta^{\prime}\right)$ corresponds to the cover transformation $\gamma(z)=$ $z+1$ of $U$, and let $\tilde{f}: \omega^{-1}(V) \rightarrow V_{p}$ be a map of holomorphic families that satisfies (4.4). The induced holomorphic map $f: U \rightarrow T_{p}$ then satisfies

$$
f(z+1)=\mu(\gamma)(f(z)) \text { for all } z \in U .
$$

If $\mu(\gamma)$ is given by equation (7.1), then $\Phi \circ f: U \rightarrow U^{3 p-3}$ satisfies

$$
(\Phi \circ f)(z+1)=(\Phi \circ f)(z)+\sum_{j=1}^{k} n_{j} e_{j}
$$

The conclusion that each $n_{j} \geq 0$ therefore follows at once from the following elementary fact.

LEMMA 7.1. Let $\varphi: U \rightarrow U$ be a holomorphic function. Suppose there is an integer $n$ such that

$$
\varphi(z+1)=\varphi(z)+n \text { for all } z \in U .
$$

Then $n \geq 0$.

Proof. By hypothesis there is a holomorphic function $\psi: \Delta^{\prime} \rightarrow \Delta^{\prime}$ such that $\psi \circ \omega=\omega \circ \varphi: U \rightarrow \Delta^{\prime}$. Since $\psi$ is bounded, it has a holomorphic extension (still called $\psi$ ) that maps $\Delta$ into itself. Let $m(\geq 0)$ be the order of vanishing of $\psi$ at the origin. Fix $r$ in $(0,1)$ and $c$ in $U$ so that $\omega(c)=r$. Then

$$
\begin{aligned}
0 \leq m & =\frac{1}{2 \pi i} \int_{|\zeta|=r} \frac{\psi^{\prime}(\zeta) d \zeta}{\psi(\zeta)} \\
& =\frac{1}{2 \pi i} \int_{z=c}^{c+1} \frac{\psi^{\prime}(\omega(z)) \omega^{\prime}(z) d z}{\psi(\omega(z))}=\varphi(c+1)-\varphi(c)=n .
\end{aligned}
$$

That proves the lemma and the theorem.

REMARKS. (1) Theorem 3 places restrictions on the monodromy of any family $\pi: V \rightarrow \Delta^{\prime}$ whose monodromy group has infinite order. Imayoshi [10] has shown that every such family has a central fiber, which is the quotient of a noded Riemann surface by a (finite cyclic) group of automorphisms. This noded Riemann surface is obtained 
topologically by pinching a set of homotopically independent simple loops on $S$. (For instance, in the examples of $\S 2$ the central fiber is $X \cup Y$ with $P_{1}$ and $P_{2}$ attached respectively to $Q_{1}$ and $Q_{2}$. The pinched loops are the curves $A_{2}$ and $D_{2}$ in Figure 1c.) Let $\tau_{1}, \ldots, \tau_{k}$ in $\operatorname{Mod}_{p}$ correspond to the negative Dehn twists about these pinched loops.

According to [10, p. 297] some positive power $\rho$ of $\mu(\gamma)$ satisfies

$$
\mu\left(\gamma^{\rho}\right)=\mu(\gamma)^{\rho}=\tau_{1}^{n_{1}} \cdots \tau_{k}^{n_{k}} .
$$

Applying Theorem 3 to the pullback of $\pi: V \rightarrow \Delta^{\prime}$ by the map $z \mapsto z^{\rho}$ of $\Delta^{\prime}$ onto itself, we conclude that $n_{j} \geq 0$ for all $j$. This condition in fact characterizes infinite monodromy groups of families over $\Delta^{\prime}$. Let $\alpha \in \operatorname{Mod}_{p}$ be an element of infinite order. In our forthcoming paper [7] we prove that there is a holomorphic family $\pi: V \rightarrow \Delta^{\prime}$ with $\mu(\gamma)=\alpha$ if and only if some positive power $\alpha^{\rho}$ is a product of positive powers of negative Dehn twists about a set of homotopically independent loops on $S$.

(2) The special coordinates used in the proof of Theorem 3 have the additional property

$$
U^{3 p-3}+c \subset \Phi\left(T_{p}\right) \text { for some } c \in U^{3 p-3} \text {. }
$$

That property makes possible a more formal proof of the sufficiency of (7.1). Define $f: U \rightarrow T_{p}$ by putting

$$
f(z)=\Phi^{-1}\left(2 c+z \sum_{j=1}^{k} n_{j} e_{j}\right) \quad \text { for all } z \in U .
$$

Then $f(z+1)=\mu(\gamma)(f(z))$, and we can construct a family over $\Delta^{\prime}$ with the required monodromy as a quotient of the pullback of the Teichmüller curve by $f$. (See Theorem 4.1 of [9] or Theorem 7.1 of [3].)

8. Proof of Theorem 2. We use the notations of $\S \S 4$ and 5. Recall that $\pi^{\#}: V^{\#} \rightarrow B^{\#}$ is the pullback of $\pi: V \rightarrow B$ by the holomorphic universal covering map $\omega: B^{\#} \rightarrow B$. The action (4.3) of $\Gamma(=$ $\pi_{1}\left(B, b_{0}\right)$ ) on $V^{\#}$ induces the usual pullback action on line bundles $L^{\#}$ over $V^{\#}$ :

$$
\gamma \cdot L^{\#}=\left(\tilde{\gamma}^{-1}\right)^{*}\left(L^{\#}\right) \text { for all } \gamma \in \Gamma .
$$

Since $\left(\tilde{\gamma}^{-1}\right)^{*} K_{\mathrm{rel}}\left(V^{\#}\right)$ and $K_{\mathrm{rel}}\left(V^{\#}\right)$ are equivalent and pullbacks preserve tensor products, $(8.1)$ determines an action of $\Gamma$ on the set $F_{n}^{\#}$ of equivalence classes of $n$th roots of $K_{\text {rel }}\left(V^{\#}\right)$ for each $n \geq 2$. 
Let $\tilde{\omega}: V^{\#} \rightarrow V$ be the map $\tilde{\omega}(t, v)=v$. (Recall that $V^{\#}$ is defined by equation (4.2).) We need a routine preliminary result.

LEMMA 8.1. If $L$ is an $n$th root of $K_{\mathrm{rel}}(V)$, then $L^{\#}=\tilde{\omega}^{*}(L)$ is an $n$th root of $K_{\mathrm{rel}}\left(V^{\#}\right)$, and $\gamma \cdot L^{\#}$ is equivalent to $L^{\#}$ for every $\gamma$ in $\Gamma$.

Proof. $L^{\#}$ is an $n$th root of $K_{\text {rel }}\left(V^{\#}\right)$ because $K_{\text {rel }}\left(V^{\#}\right)$ and $\tilde{\omega}^{*}\left(K_{\text {rel }}(V)\right)$ are equivalent (see Proposition 1 of [15]) and pullbacks preserve tensor products. $L^{\#}$ and $\gamma \cdot L^{\#}$ are equivalent because $\tilde{\omega} \circ \tilde{\gamma}=$ $\tilde{\omega}$ for all $\gamma$ in $\Gamma$.

COROLlary 8.2. Let $F_{n}$ be the set of equivalence classes of $n$th roots of $K_{\mathrm{rel}}(V)$. The map $L \mapsto \tilde{\omega}^{*}(L)$ induces a well-defined map of $F_{n}$ into the set of elements of $F_{n}^{\#}$ that are fixed by every member of $\Gamma$.

We also need a result from [15]. For any line bundle $\pi: L \rightarrow M$, let $\pi_{0}: L_{0} \rightarrow M$ be the fiber bundle obtained by removing from $L$ the image of the zero section. (Thus $\pi_{0}: L_{0} \rightarrow M$ is the principal $\mathbb{C}^{*}$-bundle associated to $\pi: L \rightarrow M$.) Every self-equivalence $F: L \rightarrow L$ restricts to a biholomorphic map $F \mid L_{0}: L_{0} \rightarrow L_{0}$.

For each $n \geq 2$ let $\Lambda_{n}\left(L_{0}\right)$ be the set of homomorphisms $\lambda$ : $H_{1}\left(L_{0}, Z_{n}\right) \rightarrow Z_{n}$ such that $\lambda(\beta)=1$, where $\beta$ is the homology class of the positive loop around the origin in the fiber $\mathbb{C}^{*}$ of $L_{0}$.

Proposition 8.3 (Sipe [15]). Let $\pi: K \rightarrow M$ be any line bundle such that the restriction $F \mid K_{0}$ of every self-equivalence $F: K \rightarrow K$ is homotopic (in $K_{0}$ ) to the identity map of $K_{0}$. There is a natural bijective map $\lambda \mapsto L_{\lambda}$ between $\Lambda_{n}\left(K_{0}\right)$ and the set of equivalence classes of $n$th roots of $K$.

This result is proved in Section 5.1 of [15], where it is stated as Theorem 1a. In that statement $K=K_{\mathrm{rel}}\left(V_{p}\right)$ and $M=V_{p}$, but the proof in [15] caries over verbatim to the general case stated here. The natural bijection is given as follows. Each $\lambda$ in $\Lambda_{n}\left(K_{0}\right)$ determines an $n$-fold covering space of $K_{0}$. That covering space is $\left(L_{\lambda}\right)_{0}$, and the $n$-fold covering map $\left(L_{\lambda}\right)_{0} \rightarrow K_{0}$ is the map $v \mapsto v \otimes \cdots \otimes v$ from $\left(L_{\lambda}\right)_{0}$ to $\left(L_{\lambda}^{\otimes n}\right)_{0}$.

LEMMA 8.4. The map of holomorphic families $\tilde{f}: V^{\#} \rightarrow V_{p}$ in (4.4) induces a bijection between $\Lambda_{n}\left(K_{\mathrm{rel}}\left(V_{p}\right)_{0}\right)$ and $\Lambda_{n}\left(K_{\mathrm{rel}}\left(V^{\#}\right)_{0}\right)$. 
Proof. The map $\tilde{f}$ determines a bundle map $g: K_{\text {rel }}\left(V^{\#}\right) \rightarrow K_{\text {rel }}\left(V_{p}\right)$ such that the diagram

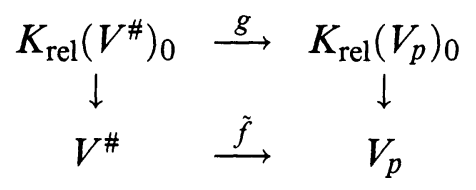

commutes (cf. Proposition 1 of [17]). Let $g_{*}: H_{1}\left(K_{\mathrm{rel}}\left(V^{\#}\right)_{0}, Z_{n}\right) \rightarrow$ $H_{1}\left(K_{\text {rel }}\left(V_{p}\right)_{0}, Z_{n}\right)$ be the induced map of homology groups. Since $g$ maps fibers to fibers isomorphically, it is obvious that $g_{*}(\beta)=\beta$ and that $\lambda \mapsto \lambda \circ g_{*}$ maps $\Lambda_{n}\left(K_{\text {rel }}\left(V_{p}\right)_{0}\right)$ into $\Lambda_{n}\left(K_{\text {rel }}\left(V^{\#}\right)_{0}\right)$. To see that this map is bijective, we shall prove that $g$ induces an isomorphism of fundamental groups.

The exact homotopy sequences of the fibrations in (8.2) produce a commutative diagram

$$
\begin{aligned}
& \rightarrow \pi_{2}\left(V^{\#}\right) \rightarrow \pi_{1}\left(\mathbb{C}^{*}\right) \stackrel{l_{*}}{\longrightarrow} \pi_{1}\left(K_{\mathrm{rel}}\left(V^{\#}\right)_{0}\right) \rightarrow \pi_{1}\left(V^{\#}\right) \rightarrow 1 \\
& \text { id } \downarrow \quad g_{*} \downarrow \\
& \rightarrow \pi_{2}\left(V_{p}\right) \rightarrow \pi_{1}\left(\mathbb{C}^{*}\right) \stackrel{g_{*} l_{*}}{\longrightarrow} \pi_{1}\left(K_{\mathrm{rel}}\left(V_{p}\right)_{0}\right) \rightarrow \pi_{1}\left(V_{p}\right) \rightarrow 1
\end{aligned}
$$

with exact rows. Here $l_{*}$ is induced by the inclusion map $l$ from the fiber $\mathbb{C}^{*}$ to $K_{\text {rel }}\left(V^{\#}\right)_{0}$. We know that $T_{p}$ is contractible and $V_{p}$ is diffeomorphic to $T_{p} \times S$. Therefore $\pi_{2}\left(V_{p}\right)=1, g_{*} l_{*}$ is injective, and we have a commutative diagram

$$
\begin{aligned}
& 1 \rightarrow \pi_{1}\left(\mathbb{C}^{*}\right) \rightarrow \pi_{1}\left(K_{\mathrm{rel}}\left(V^{\#}\right)_{0}\right) \rightarrow \pi_{1}\left(V^{\#}\right) \rightarrow 1 \\
& \text { id } \downarrow \quad g_{*} \downarrow \\
& 1 \rightarrow \pi_{1}\left(\mathbb{C}^{*}\right) \rightarrow \pi_{1}\left(K_{\text {rel }}\left(V_{p}\right)_{0}\right) \rightarrow \pi_{1}\left(V_{p}\right) \rightarrow 1
\end{aligned}
$$

whose rows are short exact sequences. Since $\pi^{\#}: V^{\#} \rightarrow B^{\#}$ is (equivalent to) the pullback of the Teichmüller curve by $f: B^{\#} \rightarrow T_{p}, V^{\#}$ is diffeomorphic to $B^{\#} \times S$ and $\tilde{f}_{*}: \pi_{1}\left(V^{\#}\right) \rightarrow \pi_{1}\left(V_{p}\right)$ is an isomorphism. Hence so is $g_{*}$.

Recall that $E_{n}$ is the set of equivalence classes of $n$th roots of $K_{\text {rel }}\left(V_{p}\right)$. The action of the monodromy group on $E_{n}$ produces an action of $\Gamma$; we put

$$
\gamma \cdot[L]=\mu(\gamma) \cdot[L]
$$

if $\gamma \in \Gamma$ and $[L]$ is the equivalence class of an $n$th root $L$ of $K_{\text {rel }}\left(V_{p}\right)$.

LEMMA 8.5. The map of holomorphic families $\tilde{f}: V^{\#} \rightarrow V_{p}$ induces the $\Gamma$-equivariant bijective map $[L] \mapsto\left[\tilde{f}^{*}(L)\right]$ from $E_{n}$ to $F_{n}^{\#}$. 
Proof. Since $B^{\#}$ and $T_{p}$ are simply connected, the relative canonical bundles of the families $V^{\#} \rightarrow B^{\#}$ and $V_{p} \rightarrow T_{p}$ satisfy the hypothesis of Proposition 8.3 (cf. $\S 2.3$ of [15]). Therefore the bijections of Proposition 8.3 and Lemma 8.4 establish a bijection between $E_{n}$ and $F_{n}^{\#}$. Explicitly, $[L]$ in $E_{n}$ is represented by $L_{\lambda}$ for some $\lambda$ in $\Lambda_{n}\left(K_{\text {rel }}\left(V_{p}\right)_{0}\right)$, and its image under the bijection is $\left[L_{\lambda \circ g_{*}}\right]$ in $F_{n}^{\#}$. To see that the bijection is given by pullback, we shall verify that $\tilde{f}^{*}\left(L_{\lambda}\right)$ is equivalent to $L_{\lambda \circ g_{*}}$. The definition of the covering spaces $\left(L_{\lambda \circ g_{*}}\right)_{0}$ and $\left(L_{\lambda}\right)_{0}$ shows that the map $g$ in (8.2) lifts to a map $\tilde{g}$ from $\left(L_{\lambda \circ g_{*}}\right)_{0}$ to $\left(L_{\lambda}\right)_{0}$, producing a commutative diagram

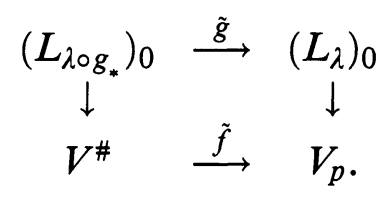

Since $\tilde{g}$ is holomorphic and maps fibers to fibers biholomorphically (cf. $\S 5.1$ of [15]), it extends to a bundle map that covers $\tilde{f}$. Therefore $L_{\lambda \circ g_{*}} \cong \tilde{f}^{*}\left(L_{\lambda}\right) \cong \tilde{f}^{*}(L)$, as we claimed.

Finally, the $\Gamma$-equivariance follows from (4.4), since

$$
\tilde{\gamma}^{*}\left(\tilde{f}^{*}(L)\right) \cong(\tilde{f} \circ \tilde{\gamma})^{*}(L)=(\mu(\gamma) \circ \tilde{f})^{*}(L) \cong \tilde{f}^{*}\left(\mu(\gamma)^{*}(L)\right)
$$

for all $\gamma$ in $\Gamma$.

Proof of Theorem 2. We must define a map from $F_{n}$ to the subset of $E_{n}$ on which $\Gamma$ acts trivially by (8.3). The required map is obtained by composing the map from $F_{n}$ to $F_{n}^{\#}$ defined in Corollary 8.2 with the inverse of the bijective map from $E_{n}$ to $F_{n}^{\#}$ defined in Lemma 8.5 .

REMARK. It is not hard to prove that the map in Corollary 8.2 from $F_{n}$ to the subset of $F_{n}^{\#}$ on which $\Gamma$ acts trivially is bijective if $B=\Delta^{\prime}$ or any noncompact Riemann surface. Neither injectivity nor surjectivity holds in general. Injectivity fails if there are nontrivial flat line bundles of order $n$ over the base space $B$. For example, suppose $B$ is a compact Riemann surface of genus $p \geq 2, V=B \times B$, and $\pi: V \rightarrow B$ is projection onto the first factor. If $K$ is the canonical bundle of $B$, then $K_{\text {rel }}(V) \cong B \times K$ and Proposition 8.3 shows that $F_{n}$ is much larger than $F_{n}^{\#}$ for any $n \geq 2$ that divides $2 p-2$. We shall give examples of nonsurjectivity in a sequel to this paper. We are grateful to Claude LeBrun for bringing the question of surjectivity to our attention. 


\section{REFERENCES}

[1] M. F. Atiyah, Riemann surfaces and spin structures, Ann. Scient. Éc. Norm. Sup., 4e série, 4 (1971), 47-62.

[2] L. Bers, Finite-dimensional Teichmüller spaces and generalizations, Bull. Amer. Math. Soc., 5 (1981), 131-172.

[3] C. J. Earle, Families of Riemann surfaces and Jacobi varieties, Ann. of Math., 107 (1978), 255-286.

[4] _- Some explicit real analytic trivializations of the Teichmüller curves, Proc. Amer. Math. Soc., 104 (1988), 503-506.

[5] C. J. Earle and R. S. Fowler, Holomorphic families of open Riemann surfaces, Math. Ann., 270 (1985), 249-273.

[6] C. J. Earle, and A. Marden, Geometric complex coordinates for Teichmüller space, to appear.

[7] C. J. Earle and P. L. Sipe, Invariant Teichmüller disks and the monodromy of holomorphic families, to appear.

[8] J. D. Fay, Theta functions on Riemann surfaces, Lecture Notes in Mathematics, No. 352, Springer-Verlag, 1973.

[9] A. Grothendieck, Techniques de construction en géométrie analytique, Séminaire H. Cartan, 13ème année: 1960/61, Exp. 7.

[10] Y. Imayoshi, Holomorphic families of Riemann surfaces and Teichmüller spaces. In "Riemann surfaces and related topics: Proceedings of the 1978 Stony Brook conference", I. Kra, and B. Maskit, eds., Ann. Math. Studies, No. 97, pp. 277300, Princeton University Press, 1981.

[11] I. Kra, Canonical mappings between Teichmüller spaces, Bull. Amer. Math. Soc., 4 (1981), 143-179.

[12] __ Horocyclic coordinates for Riemann surfaces and moduli spaces, I: Teichmüller and Riemann spaces of Kleinian groups, J. Amer. Math. Soc., 3 (1990), 499-578.

[13] B. Maskit, Moduli of marked Riemann surfaces, Bull. Amer. Math. Soc., 80 (1974), 773-778.

[14] S. Nag, The Complex Analytic Theory of Teichmüller Spaces, Wiley-Interscience, John Wiley and Sons, New York, 1988.

[15] P. L. Sipe, Roots of the canonical bundle of the universal Teichmüller curve and certain subgroups of the mapping class group, Math. Ann., 260 (1982), 67-92.

[16] __ Some finite quotients of the mapping class group of a surface, Proc. Amer. Math. Soc., 97 (1986), 515-524.

[17] _ Families of compact Riemann surfaces which do not admit nth roots. In "Holomorphic functions and moduli, Vol. 2", MSRI Publications 11, pp. 285290, Springer-Verlag, 1988.

Received December 13, 1988 and in revised form August 14, 1990. This research was partly supported by NSF grant DMS-8601016 (first author) and by a Fulbright Research Grant (second author).

CORNELl UNIVERSITY

ITHACA, NY 14853

AND 


\section{PACIFIC JOURNAL OF MATHEMATICS EDITORS}

\author{
V. S. VARADARAJAN \\ (Managing Editor) \\ -University of California \\ Los Angeles, CA 90024-1555-05 \\ Herbert Clemens \\ University of Utah \\ Salt Lake City, UT 84112 \\ THOMAS ENRIGHT \\ University of California, San Diego \\ La Jolla, CA 92093
}

R. FINN

Stanford University

Stanford, CA 94305

Hermann FlaschKa

University of Arizona

Tucson, AZ 85721

VAUGHAN F. R. JONES

University of California

Berkeley, CA 94720

SteVen KerCKhoff

Stanford University

Stanford, CA 94305
C. C. MOORE

University of California

Berkeley, CA 94720

Martin ScharlemanN

University of California

Santa Barbara, CA 93106

\section{HAROLD STARK}

University of California, San Diego

La Jolla, CA 92093

\section{ASSOCIATE EDITORS}

\begin{tabular}{|c|c|c|c|c|}
\hline R. ARENS & $\begin{array}{l}\text { E. F. BECKENBACH } \\
(1906-1982)\end{array}$ & NeUManN & $\begin{array}{l}\text { F. WoLF } \\
(1904-1989)\end{array}$ & K. YoshidA \\
\hline \multicolumn{5}{|c|}{ SUPPORTING INSTITUTIONS } \\
\hline \multicolumn{2}{|c|}{ UNIVERSITY OF ARIZONA } & \multicolumn{3}{|c|}{ UNIVERSITY OF OREGON } \\
\hline \multirow{2}{*}{\multicolumn{2}{|c|}{$\begin{array}{l}\text { UNIVERSITY OF BRITISH COLUMBIA } \\
\text { CALIFORNIA INSTITUTE OF TECHNOLOGY }\end{array}$}} & \multicolumn{3}{|c|}{ UNIVERSITY OF SOUTHERN CALIFORNIA } \\
\hline & & \multicolumn{3}{|c|}{ STANFORD UNIVERSITY } \\
\hline CALIFORNIA INSTITUTE OF TECHNOLOGY & UNIVERSITY OF CALIFORNIA & \multicolumn{3}{|c|}{ UNIVERSITY OF HAWAII } \\
\hline \multicolumn{2}{|c|}{ MONTANA STATE UNIVERSITY } & \multicolumn{3}{|c|}{ UNIVERSITY OF TOKYO } \\
\hline \multicolumn{2}{|c|}{ UNIVERSITY OF NEVADA, RENO } & \multicolumn{3}{|c|}{ UNIVERSITY OF UTAH } \\
\hline \multicolumn{2}{|c|}{ NEW MEXICO STATE UNIVERSITY } & \multicolumn{3}{|c|}{ WASHINGTON STATE UNIVERSITY } \\
\hline OREGON STA & UNIVERSITY & UNIVERS & OF WASHING & \\
\hline
\end{tabular}




\section{Pacific Journal of Mathematics}

Vol. 150, No. $1 \quad$ September, 1991

Jan Aarts, Charles Lemuel Hagopian and Lex Gerard Oversteegen, The orientability of matchbox manifolds $\ldots \ldots \ldots \ldots \ldots \ldots \ldots \ldots \ldots \ldots \ldots \ldots$

Roger W. Barnard, Carl Hanson Fitzgerald and Sheng Gong, The growth and $1 / 4$-theorems for starlike mappings in $\mathbf{C}^{n} \ldots \ldots \ldots \ldots \ldots \ldots \ldots$

Allan Berele, Trace rings for verbally prime algebras $\ldots \ldots \ldots \ldots \ldots \ldots \ldots 23$

Quo-Shin Chi, Curvature characterization and classification of rank-one

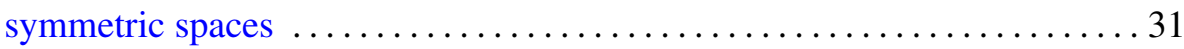

Harold Gerard Donnelly and Jeffrey Marc Lee, Domains in Riemannian

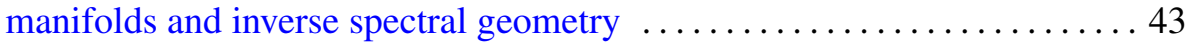

Clifford John Earle, Jr. and Patricia Lilaine Sipe, Families of Riemann

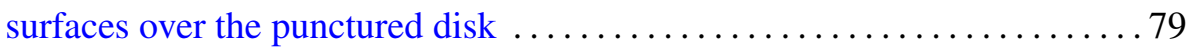

Mark P. Hughes, Dihedral group actions on homotopy complex projective

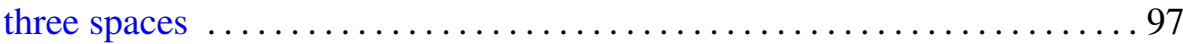

Robert F. Lax and Carl Widland, Gap sequences at a singularity ........111

Takahiko Nakazi, Bounded Hankel forms with weighted norms and lifting

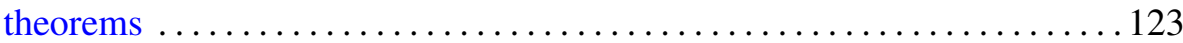

Douglas Murray Pickrell, Mackey analysis of infinite classical motion groups

Martha Rzedowski-Calderón and Gabriel Daniel Villa-Salvador,

Automorphisms of congruence function fields 167

Peter N-S Wong, Equivariant Nielsen fixed point theory for $G$-maps 